\title{
Model reference control of conceptual clutched train substituted for vehicular friction clutch
}

\author{
Wei Huang ${ }^{1, a}$, Pakharuddin Mohd Samin ${ }^{1}$ and Kamarul Baharin Tawi ${ }^{1}$ \\ ${ }^{1}$ Department of Aeronautical, Automotive and Ocean Engineering, Universiti Teknologi Malaysia, 81310 Johor, Malaysia
}

\begin{abstract}
In order to essentially reduce heat generation and frictional dissipation carried by friction clutch engagement, conceptual design of clutched train combined with hydrostatic braking system is proposed as a novel substitution for vehicular friction clutch. Potential collateral merits of clutched train may improve service life and control accuracy since less friction heat generated during synchronization process. Parameter of clutched train is obtained by Genetic Algorithm optimization aiming at axial space-saving and light weight. Control-oriented model of proposed concept is derived and used in Model Reference Control development. Based on optimum parameter of clutched train, simulation result has shown the functionality of clutched train on vehicle standing-start, and well-behaved Model Reference Control on smoothing clutched train synchronization process.
\end{abstract}

\section{Introduction}

Friction clutch is known as a key mechanical connection and disconnection between an engine and a transmission in a car. The basic function of clutch is supposed to transfer power from engine to vehicle with desired smoothly and efficiently. However, owing to it transmitting torque through friction, some issues particularly in terms of friction dissipation [1] and shift comfort [2-4] are inevitable. For example in case when significant heat generation during slipping phase, high nonlinear fluctuation of friction coefficient [3, 4] would make it difficult to give an accurate signal to actuator for the estimation of transmitted clutch torque.

As a branch of planetary gearset application, concept of clutched train with hydraulic system is proposed in this paper to substitute for friction clutch used in a car and mated to automated mechanical transmission to ensure smooth transfer of torque with a little heat generated by gear-meshed sliding friction [5]. Note that less heating is associated with improving mechanical life, as well as more accurate control due to little variation on friction coefficient. Clutched train utilizes the alternation between one degree-of-freedom (DOF) and two DOFs of rotary motion to determine the output instead of friction. The primary advantages of proposed concept lie in avoiding high non-linearity and significant frictional dissipation with engagement, and in return improving the control veracity in practice.

This paper would present the conceptual design and application of Model Reference Control (MRC) algorithm on clutched train synchronization process control. Simulation results show that the functionality of conceptual design is to connect and disconnect engine and transmission, and the designed MRC could work with conceptual design to reduce vehicle jerk at vehicle standing-start.

\section{Conceptual design}

As shown in Figure 1, the configuration of conceptual design is comprised of a symmetrical compound planetary gearset and a hydraulic system. The compound planetary gearset can be considered as a combination of single stage Planetary-Gearset-I (PGS-I) and PlanetaryGearset-II (PGS-II), which have the same parameters and share one arm. The PGS-I ring gear is fixed. The PGS-I sun gear is coupled with the driving shaft, which is driven by the output shaft of an engine. The PGS-II sun gear is coupled to a coaxial output shaft which drives an automatic transmission.

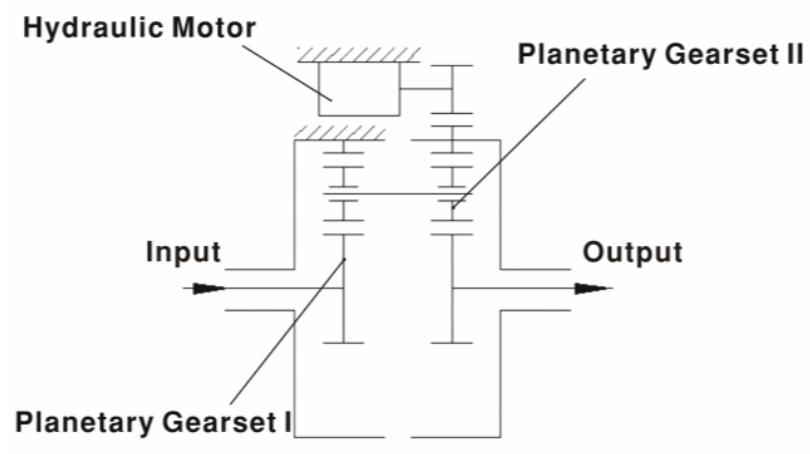

Figure 1. Schematic structure of clutched train.

\footnotetext{
${ }^{\mathrm{a}}$ Corresponding author: huangweiamoy@gmail.com
} 
Figure 2 illustrates the synchronization process of clutched train with hydrostatic braking system from the opening to sticking phase. The mode-switch valve would hand off the DOF of rotational dynamics from two to one or from one to two via controlling the reversible motorpump free or locked. In the opening and slipping mode, the mode switch is set to connect the reversible motorpump discharge port to the pressure relief valve for regulating the operating pressure. In the sticking mode, the mode-switch valve is shifted to connect the discharge port to check valve to cut the discharge flow. As an expected consequence, it is supposed to have same capability as a conventional clutch in interrupting and transferring the power from engine to transmission. The torque of output shaft during slipping phase, which depends on the back pressure of reversible motor-pump, can be controlled via the pressure relief valve. Accumulator can be installed to harvest the fluid energy produced by reversible motor-pump for decelerating to zero instead of friction clutch converting into heat. In other words, it makes the reversible motor-pump act as a pump to recover the pent-up momentum-carrying energy. Note that after synchronization the PGS-II ring gear is held by the back pressure of reversible motor-pump without any energy supply in terms of keeping engagement. Once overload is happened on clutched train, pressure relief valve can ensure clutched train working on safe operation by setting maximum allowable pressure.

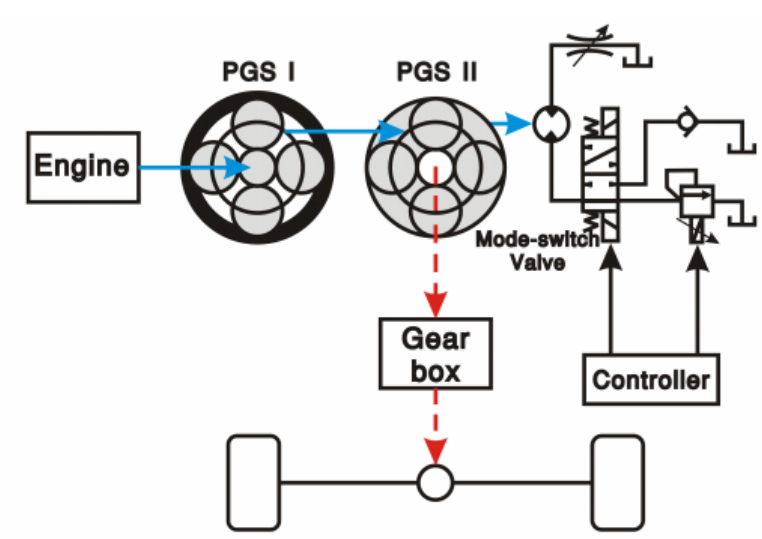

(a)

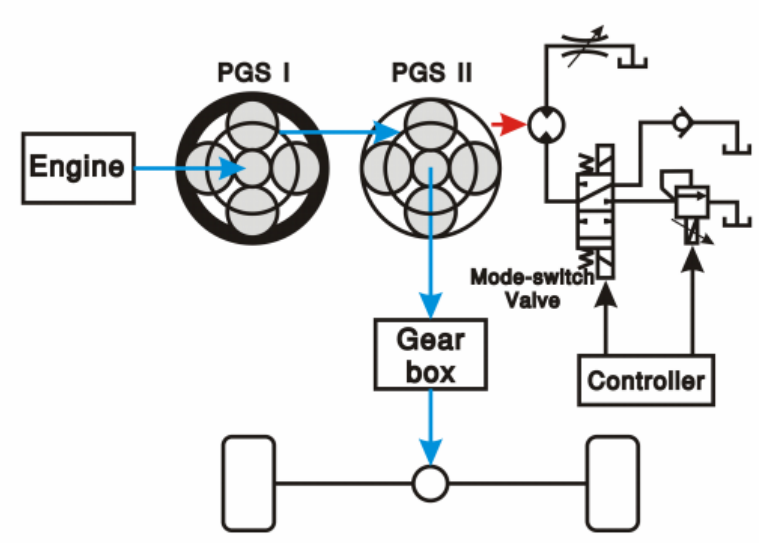

(b)
Figure 2. Power flow in driveline equipped with clutched train working in the (a) Opening phase; (b) Sticking phase (PGS-II ring gear locked).

\section{Parameter optimization}

In view of other merits about friction clutch like compact size and light weight, the parameters correlated to proposed gear train should be taken into account for compact size and light weight as well. Referring to literature[6], Genetic Algorithm (GA) was recognized as an effective and efficient technique to optimize weight design problem of gear train. Several reasonable gear train parameters are selected, and formulated for dimension optimization problem and constrained by the planetary gearset geometry limitations, mating conditions and load capacity, and solve the problem by using GA.

\subsection{GA setting}

In order to create better new individuals, GA would repeat selection, crossover and mutation until reaching the optimum solution to the problem. The main setting of GA includes:

i) Crossover: 0.85 . Determines the probability that an offspring will be created by the crossover of two parents as opposed to a replica of one parent;

ii) Mutation: 0.1. Determines the probability of a spot mutation occurring to each allele in a new population;

iii) Population Size: 400. The population size determines the number of chromosomes that make up a generation.

\subsection{Optimum parameter}

The optimization result of gear train parameters is listed in Table 1 below. These optimum parameters are used to investigate the functionality and effects on vehicle driving comfort performance of clutched train via simulation.

Table 1. GA optimum parameter.

\begin{tabular}{clc}
\hline & \multicolumn{1}{c}{ Parameter } & Value \\
\hline$z_{S}$ & Teeth number of sun gear & 30 \\
$b$ & Gear face width $(\mathrm{mm})$ & 10 \\
$m$ & Gear module $(\mathrm{mm})$ & 2 \\
$K$ & Gear ratio between ring gear and sun gear & 3 \\
\hline
\end{tabular}

\section{Dynamic modeling of clutched train}

\subsection{Kinematics of clutched train}

In this study, the compound planetary gearset is divided into two single stage planetary gearsets which have the same parameters and share one arm. The relationship of angular velocity among sun gear, ring gear and arm in single stage planetary gearset can be determined by the known Willis rule: 'the ratio of the relative (in regard to the arm) angular velocities is equal to their gear ratio' [7]. 
For the sun gears, ring gears and arm with the PGS-I and PGS-II, these following equations are obtained:

$$
\begin{gathered}
\omega_{S 1}+K \cdot \omega_{R 1}-(1+K) \cdot \omega_{A}=0 \\
\omega_{S 2}+K \cdot \omega_{R 2}-(1+K) \cdot \omega_{A}=0 \\
\omega_{R 1}=0
\end{gathered}
$$

where, $\omega_{S 1}$ is the angular velocity of PGS-I sun gear; $\omega_{S 2}$ is the angular velocity of PGS-II sun gear; $\omega_{R I}$ is the angular velocity of PGS-I ring gear; $\omega_{R 2}$ is the angular velocity of PGS-II ring gear; $\omega_{A}$ is the angular velocity of arm; and $K$ is the gear ratio between ring gear and sun gear, namely $z_{R} / z_{S}$. Since the parameters of PGS-I and PGS-II are totally same, they have the same gear ratio $K$.

By elimination of $\omega_{A}$ from Eq. (1a) and (1b), the following kinematics equation is obtained:

$$
\omega_{R 2}=\frac{\omega_{S 1}-\omega_{S 2}}{K}
$$

Note that while the angular velocity of PGS-II ring gear $\omega_{R 2}$ decelerating to zero via hydrostatic braking system, Eq. (2) demonstrates that $\omega_{S l}=\omega_{S 2}$, namely clutched train synchronization.

\subsection{Power flow of clutched train}

Power losses in gear train (e.g. gear-meshed friction losses) usually is neglected to reasonably simplify the dynamic model on control orient with little consequence. In accordance with the law of energy preservation the sum of powers transmitted by sun gear, ring gear and arm in each single gear train must equal zero [7]:

$$
\begin{gathered}
M_{S 1} \cdot \omega_{S 1}+M_{A} \cdot \omega_{A}=0 \\
M_{A} \cdot \omega_{A}+M_{S 2} \cdot \omega_{S 2}+M_{R 2} \cdot \omega_{R 2}=0
\end{gathered}
$$

where $M_{S 1}$ is the torque of PGS-I sun gear; $M_{S 2}$ is the torque of PGS-II sun gear; $M_{R 1}$ is the torque of PGS-I ring gear; $M_{R 2}$ is the torque of PGS-II ring gear; $M_{A}$ is the torque of arm.

From the condition of equilibrium of torques, the equations are noted [7]:

$$
\begin{aligned}
& M_{S 1}+M_{R 1}+M_{A}=0 \\
& M_{S 2}+M_{R 2}+M_{A}=0
\end{aligned}
$$

Based on Eq. (1a) to (1c), (3a) to (3b) and (4a) to (4b), the relationships of torques among sun gear, ring gear and arm of the PGS-I and PGS-II are derived as follows:

$$
M_{S 1}=M_{S 2}=\frac{1}{K} M_{R 2}
$$

\section{Control-oriented model of slip-stick phase}

Since standing-start maneuver has been described as a typical manifestation of phenomenon on longitudinal oscillations excited by friction clutch engagement $[8,9]$, control algorithm for clutched train synchronization process would be vital important to vehicle driving comfort. Therefore, control-oriented model of clutched train would be built in this section.

\subsection{Slipping phase}

In light of Eq. (5), torque of sun gear of planetary gearset $M_{S}$ herein is introduced and denoted as $M_{S}=M_{S 1}=M_{S 2}$. In the slipping phase, the driving shaft's angular velocity is not equal to the driven shaft's angular velocity, namely $\omega_{e} \neq \omega_{r}$. The vehicle driveline is considered as a lumped mass system. The simplified model of clutched train on controlled orient is set up and shown in Figure 3.

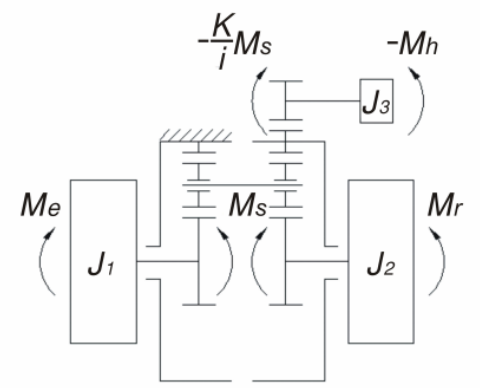

Figure 3. Simplified model of clutched train.

According to the Newton's Second Law, the simplified dynamics of clutched train in the slipping phase is given as follows:

$$
\begin{gathered}
J_{1} \cdot \dot{\omega}_{e}=M_{e}-M_{S} \\
J_{2} \cdot \dot{\omega}_{r}=M_{S}-M_{r} \\
J_{3} \cdot \dot{\omega}_{h}=\frac{K}{\mu} M_{S}-\frac{\Delta p \cdot D_{v}}{2 \pi} \eta_{m}
\end{gathered}
$$

where $J_{l}$ is the total moment of inertial of the driving shaft, including the crankshaft, flywheel and PGS-I sun gear; $J_{2}$ is the total moment of inertial of the driven shaft, including PGS-II sun gear, driveline, tires and body; $J_{3}$ is the moment of inertial of reversible motor-pump shaft; $\omega_{h}$ is the angular velocity of the reversible motor-pump; $M_{e}$ is engine output torque; $M_{r}$ is vehicle resistant torque; $\mu$ is the gear ratio between the PGS-II ring gear and the gear coupled with reversible motor-pump output shaft, namely $z_{R 2} / z_{h} ; \Delta p$ is the difference of pressure between the hydraulic motor inlet and outlet; $D_{v}$ is displacement of hydraulic motor; and $\eta_{m}$ is mechanical efficiency of hydraulic motor.

Considering the magnitude of $J_{3}$ is very small, Eq. (8) can be simplified as: 


$$
\frac{K}{\mu} M_{S} \approx \frac{\Delta p \cdot D_{v}}{2 \pi} \eta_{m}
$$

As observed from Eq. (6), (7) and (9), the value of pressure relief valve can be controlled to determine the angular velocity of PGS-II sun gear for less vehicle jerking.

\subsection{Sticking phase}

Until the angular velocity of PGS-II ring gear declines to zero under hydraulic resistance, according to Eq. (2) the driving shaft's angular velocity is equal to the driven shaft's angular velocity, namely $\omega_{e}=\omega_{r}$. Then the modeswitch valve will be shifted to sticking operation position. This will allow the PGS-II ring gear to be locked and keep $\omega_{R 2}=0$. When $\omega_{e}=\omega_{r}=\omega_{m}$, the dynamic equation can be rewritten by combining Eq. (6) and (7):

$$
\left(J_{1}+J_{2}\right) \dot{\omega}_{m}=M_{e}-M_{r}
$$

\section{Model reference control}

MRC has been proposed by Chen et al. [8] in friction clutch engagement control to compensate discontinuity of slip-stick transition. However, different feedback control law (regulator) on accommodating clutched train synchronization process is presented. The architecture of MRC including reference model and plant is established in Figure 4.

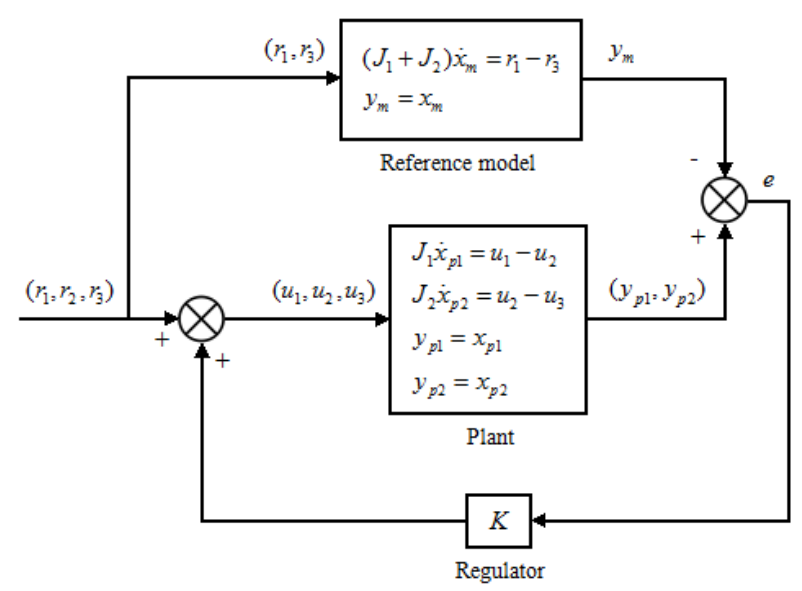

Figure 4. Architecture of MRC.

For the plant, the following variables are introduced and denoted as:

$$
\begin{gathered}
x_{p 1}=\omega_{e} ; x_{p 2}=\omega_{r} ; \\
u_{1}=M_{e} ; u_{2}=M_{S} ; u_{3}=M_{r}
\end{gathered}
$$

Substituting to Eq. (6) and (7), the state equations can be obtained:

$$
\begin{aligned}
& J_{1} \dot{x}_{p 1}=u_{1}-u_{2} \\
& J_{2} \dot{x}_{p 2}=u_{2}-u_{3}
\end{aligned}
$$

$$
\begin{aligned}
& y_{p 1}=x_{p 1} \\
& y_{p 2}=x_{p 2}
\end{aligned}
$$

For the reference model, the following variables are introduced and denoted as:

$$
x_{m}=\omega_{m}
$$

The output of the reference model are denoted as

$$
y_{m}=x_{m}
$$

The state equation is given according to Eq. (7):

$$
\left(J_{1}+J_{2}\right) \dot{x}_{m}=r_{1}-r_{3}
$$

In the proposed MRC scheme, we define that:

i) $r_{l}(t), r_{2}(t), r_{3}(t)$ are reference inputs;

ii) $\mathbf{K}$ represents the gain for the feedback regulator;

iii) $e_{1}(t)$ and $e_{2}(t)$ are two outputs of interest from the closed-loop system, given as:

$$
\begin{aligned}
& e_{1}=y_{p 1}-y_{m} \\
& e_{2}=y_{p 2}-y_{m}
\end{aligned}
$$

The proposed output feedback control algorithm is given as follows:

$$
\begin{aligned}
& u_{1}=r_{1}+k_{11} e_{1}+k_{12} e_{2} \\
& u_{2}=r_{2}+k_{21} e_{1}+k_{22} e_{2} \\
& u_{3}=r_{3}+k_{31} e_{1}+k_{32} e_{2}
\end{aligned}
$$

Then time derivative of the error vector $\mathbf{e}$ is written as

$$
\begin{aligned}
& \left(\begin{array}{l}
\dot{e}_{1} \\
\dot{e}_{2}
\end{array}\right)=\left(\begin{array}{l}
\dot{y}_{p 1}-\dot{y}_{m} \\
\dot{y}_{p 2}-\dot{y}_{m}
\end{array}\right)=\left(\begin{array}{l}
\frac{u_{1}-u_{2}}{J_{1}}-\frac{r_{1}-r_{3}}{J_{1}+J_{2}} \\
\frac{u_{2}-u_{3}}{J_{2}}-\frac{r_{1}-r_{3}}{J_{1}+J_{2}}
\end{array}\right) \\
& =\left(\begin{array}{ll}
\frac{\left(r_{1}+k_{11} e_{1}+k_{12} e_{2}\right)-\left(r_{2}+k_{21} e_{1}+k_{22} e_{2}\right)}{J_{1}}-\frac{r_{1}-r_{3}}{J_{1}+J_{2}} \\
\frac{\left(r_{2}+k_{21} e_{1}+k_{22} e_{2}\right)-\left(r_{3}+k_{31} e_{1}+k_{32} e_{2}\right)}{J_{2}}-\frac{r_{1}-r_{3}}{J_{1}+J_{2}}
\end{array}\right) \\
& =\left(\begin{array}{ll}
\frac{k_{11}-k_{21}}{J_{1}} & \frac{k_{12}-k_{22}}{J_{1}} \\
\frac{k_{21}-k_{31}}{J_{2}} & \frac{k_{22}-k_{32}}{J_{2}}
\end{array}\right)\left(\begin{array}{l}
e_{1} \\
e_{2}
\end{array}\right)+\left(\begin{array}{ccc}
\frac{1}{J_{1}}-\frac{1}{J_{1}+J_{2}} & -\frac{1}{J_{1}} & \frac{1}{J_{1}+J_{2}} \\
-\frac{1}{J_{1}+J_{2}} & \frac{1}{J_{2}} & \frac{1}{J_{1}+J_{2}}-\frac{1}{J_{2}}
\end{array}\right)\left(\begin{array}{l}
r_{1} \\
r_{2} \\
r_{3}
\end{array}\right) \\
& =\mathbf{A}\left(\begin{array}{l}
e_{1} \\
e_{2}
\end{array}\right)+\mathbf{B}
\end{aligned}
$$

It is marked that:

$$
\mathbf{A}=\left(\begin{array}{ll}
\left(\frac{k_{11}-k_{21}}{J_{1}}\right) & \left(\frac{k_{12}-k_{22}}{J_{1}}\right) \\
\left(\frac{k_{21}-k_{31}}{J_{2}}\right) & \left(\frac{k_{22}-k_{32}}{J_{2}}\right)
\end{array}\right)
$$




$$
\mathbf{B}\left(r_{1}, r_{2}, r_{3}\right)=\left(\begin{array}{ccc}
\left(\frac{1}{J_{1}}-\frac{1}{J_{1}+J_{2}}\right) & \left(-\frac{1}{J_{1}}\right) & \left(\frac{1}{J_{1}+J_{2}}\right) \\
\left(-\frac{1}{J_{1}+J_{2}}\right) & \left(\frac{1}{J_{2}}\right) & \left(\frac{1}{J_{1}+J_{2}}-\frac{1}{J_{2}}\right)
\end{array}\right)\left(\begin{array}{l}
r_{1} \\
r_{2} \\
r_{3}
\end{array}\right)
$$

Input $u_{I}$ physically stands for the engine output torque $M_{e}$, which is determined by the work load and can reflect the driver intention. Hence, $u_{l}$ is treated as a predefined input and need not be adjusted by MRC. Therefore,

$$
k_{11}=0 \& k_{12}=0
$$

Similarly, input $u_{3}$ physically stands for the resistant torque $M_{r}$ imposed on the driven shaft, which cannot be changed subjectively. Hence,

$$
k_{31}=0 \& k_{32}=0
$$

So,

$$
\mathbf{A}=\left(\begin{array}{cc}
\left(\frac{-k_{21}}{J_{1}}\right) & \left(\frac{-k_{22}}{J_{1}}\right) \\
\left(\frac{k_{21}}{J_{2}}\right) & \left(\frac{k_{22}}{J_{2}}\right)
\end{array}\right)
$$

The critical variable is $u_{2}$. In order to achieve asymptotic stability, every eigenvalue of $\mathbf{A}$ should have a strictly negative real part in accordance with Lyapunov stability theory [10]. Then

$$
\begin{gathered}
|\lambda \mathbf{I}-\mathbf{A}|=0 \\
\left|\begin{array}{cc}
\left(\lambda+\frac{k_{21}}{J_{1}}\right) & \left(\frac{k_{22}}{J_{1}}\right) \\
\left(\frac{-k_{21}}{J_{2}}\right) & \left(\lambda-\frac{k_{22}}{J_{2}}\right)
\end{array}\right|=0 \\
\lambda^{2}+\left(\frac{k_{21}}{J_{1}}-\frac{k_{22}}{J_{2}}\right) \lambda-\frac{2 k_{21} k_{22}}{J_{1} J_{2}}=0
\end{gathered}
$$

According to Vieta's formulas, thus $k_{21}$ and $k_{22}$ should satisfy:

$$
\begin{gathered}
\lambda_{1}+\lambda_{2}=\frac{k_{22}}{J_{2}}-\frac{k_{21}}{J_{1}}<0 \\
\lambda_{1} \lambda_{2}=-\frac{2 k_{21} k_{22}}{J_{1} J_{2}}>0
\end{gathered}
$$

Regarding to simulation, $k_{21}$ and $k_{22}$ are set to 0.7 and -0.7 , respectively.

Note that these three reference inputs $r_{1}, r_{2}$ and $r_{3}$ do not affect the system stability but will influence the output response e. The main function of the feed-forward regular is to avoid disturbance on the zero-input response, so that the exogenous term $\mathbf{B}\left(r_{1}, r_{2}, r_{3}\right)$ can reinforce the error convergence, the feed-forward variables $r_{1}, r_{2}$ and $r_{3}$ are therefore subject to the constraints:

$$
\left(\frac{1}{J_{1}}-\frac{1}{J_{1}+J_{2}}\right) r_{1}+\left(-\frac{1}{J_{1}}\right) r_{2}+\left(\frac{1}{J_{1}+J_{2}}\right) r_{3}=0
$$

and

$$
\left(-\frac{1}{J_{1}+J_{2}}\right) r_{1}+\left(\frac{1}{J_{2}}\right) r_{2}+\left(\frac{1}{J_{1}+J_{2}}-\frac{1}{J_{2}}\right) r_{3}=0
$$

As a consequence, $r_{2}$ can be calculated as:

$$
r_{2}=\frac{J_{2} r_{1}+J_{1} r_{3}}{J_{1}+J_{2}}
$$

The overall MRC algorithm can be summarized as:

$$
u_{2}=\frac{J_{2} r_{1}+J_{1} r_{3}}{J_{1}+J_{2}}+k_{21} e_{1}+k_{22} e_{2}
$$

As mentioned by Chen et al. [8], $u_{2}$ should be restricted under certain rate of increase $k_{f}$ due to physical mechanism limitation, namely

$$
u_{2}^{*}=k_{f} t
$$

So, the torque of sun gear of planetary gearset $M_{S}$ is determined by

$$
\bar{u}_{2}=\min \left(u_{2}, u_{2}^{*}\right)
$$

\section{Simulation results}

Co-simulation is carried out between ITI SimulationX (as plant) and Simulink/MATLAB (as controller) on the case of vehicle standing-start. The parameters of vehicle model are given in Table 2. The engine characteristics is shown in Figure 5.

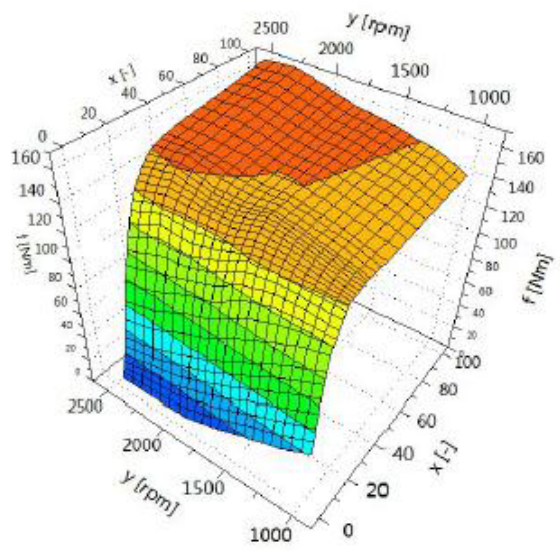

Figure 5. Engine characteristics.

The simulation results of clutched train working with MRC during slip-stick phase are shown in Figure 6, Figure 7 and Figure 8. As illustrated in Figure 6, clutched train can reach synchronization under MRC. Meanwhile, vehicle jerk observed in Figure 7 shows that it can be reduced to below $8.01 \mathrm{~m} / \mathrm{s}^{3}$ and $16.18 \mathrm{~m} / \mathrm{s}^{3}$ with less than $3 \mathrm{~Hz}$ and thus satisfy the requirement of driving comfort 
[11]. It also indicates that the simplified control-oriented model omitting the moment of inertial of reversible motor-pump shaft has little effect on comfortable synchronization performance. However, as shown in Figure 8 , the maximum of measured back pressure from reversible motor-pump are $38.89 \mathrm{MPa}$. In other words, rated pressure of hydraulic system will become the limitation of maximum transmitted torque in power train system.

Table 2. Parameters of vehicle model.

\begin{tabular}{clc}
\hline \multicolumn{1}{c}{ Parameters } & Value \\
\hline$J_{I}$ & Total moment of inertial of the driving shaft $\left(\mathrm{kg} \cdot \mathrm{m}^{2}\right)$ & 1.01 \\
$J_{2}$ & Total moment of inertial of the driven shaft $\left(\mathrm{kg} \cdot \mathrm{m}^{2}\right)$ & 0.97 \\
$J_{3}$ & Total moment of inertial of the reversible motor-pump shaft $\left(\mathrm{kg} \cdot \mathrm{m}^{2}\right)$ & 0.01 \\
$m$ & Vehicle mass $(\mathrm{kg})$ & 1400 \\
$i_{0}$ & Gear ratio of reducer & 4 \\
$i_{1}$ & Gear ratio of gearbox & 3.32 \\
$R$ & Tire radius (m) & 0.35 \\
$f$ & Rolling friction coefficient & 0.01 \\
\hline
\end{tabular}

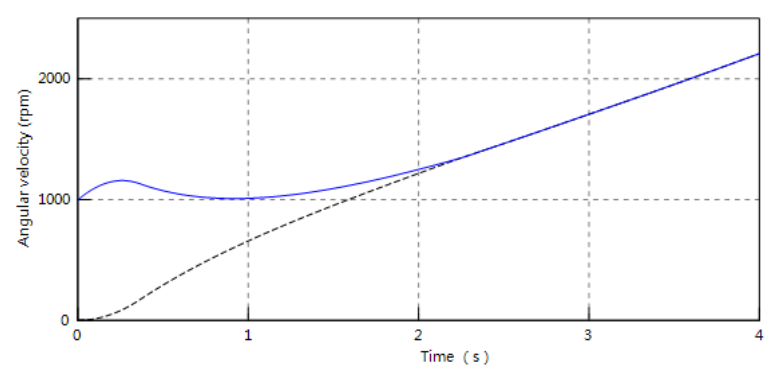

Figure 6. Angular velocity of driving and driven shaft at the slip-stick transition. (solid line: angular velocity of driving shaft, dashed line: angular velocity of driven shaft)

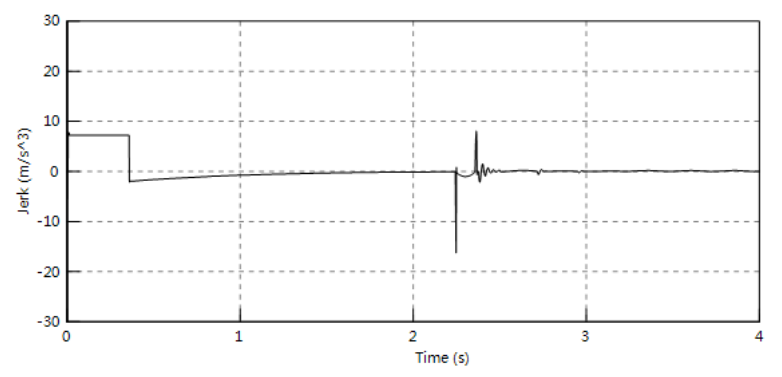

Figure 7. Derivation of vehicle acceleration, namely jerk.

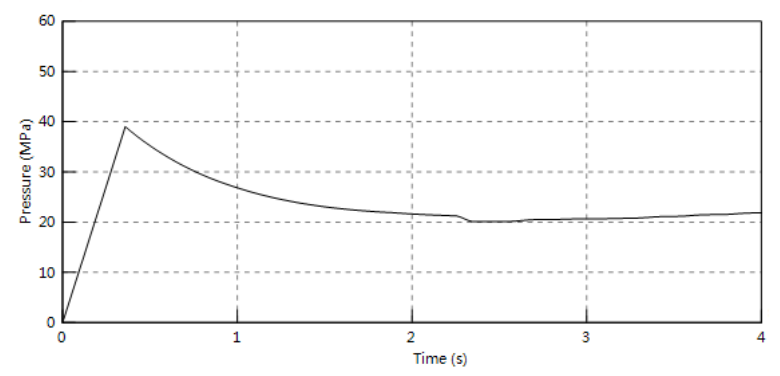

Figure 8. Pressure of hydraulic system.

\section{Conclusion}

Conceptual design of clutched train with hydrostatic braking system is provided in this paper. Control-oriented model and MRC on clutched train synchronization process are developed. Simulation results show that conceptual proposal has the desired function to connect and disconnect engine with transmission. In the period of clutched train slip-stick, vehicle jerk can be accommodated by MRC for comfortable driving experience of vehicle standing-start. However, the maximum transmitted torque of proposed concept will be primarily limited by the rated pressure of hydraulic system and result in limitation of relevant application in heavy vehicle.

\section{References}

1. V. Aravind, Engagement characteristics of a friction pad for commercial vehicle clutch system. Sadhana, 2010. 35(5): p. 585-595.

2. N. Cappetti, M. Pisaturo, A. Senatore. Modelling the cushion spring characteristic to enhance the automated dry-clutch performance: The temperature effect. P I Mech Eng D-J Aut, 2012. 0(0): p. 1-11.

3. P. Marklund, R. Larsson. Wet clutch under limited slip conditions - simplified testing and simulation. P I Mech Eng J-J Eng, 2007. 221(5): p. 545-551.

4. T. Petrun, M. Kegl, J. Flašker. Development and Validation of a Friction Model for Simulation of Friction Clutch Dynamics in a Multi-Body System, in Tribology and Design II, M. Hadfield and C.A. Brebbia, Editors. 2012, WIT Press: Southampton. p. 77-88.

5. W. Huang, P.M. Samin, K.B. Tawi, B. Supriyo. Frictional dissipation and mechanical efficiency analysis of clutched train engagement. J Appl Sci \& Agric, 2014. 9(18): p. 238-244.

6. Y. Takao, T. Takeaki, G. Mitsuo. A solution method for optimal weight design problem of the gear using genetic algorithms. Comput Ind Eng, 1998. 35(3-4): p. 523-526.

7. T. Jelaska. Gears and gear drives, 2012, John Wiley \& Sons: Chichester. 
8. L. Chen, G. Xi, C.L. Yin. Model referenced adaptive control to compensate slip-stick transition during clutch engagement. Int $\mathrm{J}$ Auto Tech-Kor, 2011. 12(6): p. 913-920.

9. D. Hrovat, W. E. Tobler. Bond graph modeling of automotive power trains. J Franklin I, 1991. 328(56): p. 623-662.

10. A. Karl W. Björn. Adaptive control, 2013, Courier Corporation: New York.

11. J. G. Zhang, Y.L. Lei, X. Hua, J. Wang, A. Ge. Proposed shift quality metrics and experimentation on AMT shift quality evaluation. in $3 r d$ International Conference on Natural Computation. 2007. 